\title{
Research on Construction of Digital Intelligent City Management System
}

\author{
Changhui Yang \\ School of Business, Zhengzhou University, 450001 \\ yang0825@126.com
}

\begin{abstract}
By means of information technology to build digital city management system can improve the level and efficiency of city management as well as the management quality of government public service. In this paper, the general structure of city management system and its subsystems have been analyzed, and then discussed the construction of the service call center of digital city management system and the basic construction principles of call center, analyzed the systematical topology framework and the function of the digital city management service call center, discusses how to design the dato interface between the related subsystem of digital city management system, it will ensure the expedite transfer between the related subsystem, and offer the timely and exact datafor city managers, that will be benefit to advance decision-making and quick response of emergency for city managers and will be provide certain lessons for the digital city management.
\end{abstract}

Keywords: Digital Intelligent City Management System, Network Topological Structure, Service call center, Data Interface

\section{Introduction}

City management is anecessary requirement for sustainable development, which is also an important measure fồ city economic and social development, meanwhile, it guarantees the process of urbanization and the establishment of the modern city. It is conducive to implement the-overall city planning and to enhance the authority of the city planning to strengthen the city management Also, it favors the normal function of city infrastructure and benefit of city investment Additionally, it makes for the improvement of the city environment, city image and degree of opening to the outside world; Apart from these, it helps enhance the comprehensive functions of cities, and promote the sustainable development of city economics and society[1]. To establish pilots before building digital city management system can accumulate valuable experience for the comprehensive construction, which can also guarantee the whole work.

The construction of digital city runs throughout the whole process of city planning, city construction, city management and service. With the construction of digital city and the use of information technology, it helps promote the transformation of government functions and the innovation of management. The innovation of system leads to the improvement of the government management and service, and then, achieves efficient service [2]. On the viewpoint of development, without the building new mode of city management and planning and managing city according to human-oriented thought, it can neither get to a new breakthrough for city management, nor create a favorable condition for the development and stability of city reform. With the system construction, the work that promotes city management to get to the target of initiative, accuracy, speediness and unification, integrates 
and optimizes the governmental information resources and the government database groups, sets up city management system covering the whole period and the whole region. To establish pilots before building digital city management system can accumulate valuable experience for the comprehensive construction, which can also guarantee the whole work [3].

\section{System Structure of Digital Intelligent City Management System}

\subsection{System Structure of Digital Intelligent City Management System}

According to the "two axis" city management mode, the digital city management system includes city management monitoring and evaluation center(i.e., supervision center of the city management), as well as the command, scheduling, coordinating center(i.e., command center of the city management). The purpose of such a setting is to separate supervision from command, each fulfills its own duties. In order to realize the new city management system, the two axis of supervision and comman should be connected seamlessly and work efficiently in a coordinate manner throughthe platform of the digital city management. Generally speaking, the digital city management system can be divided into 10 subsystems, which are shown in Figure 1.

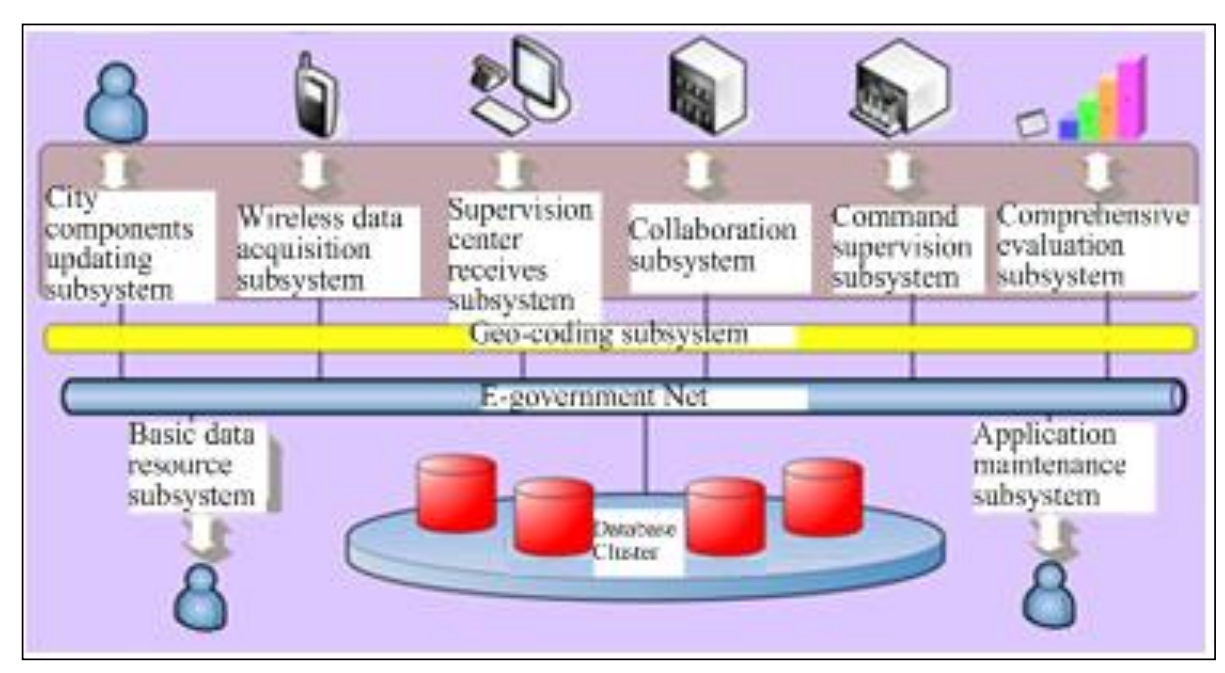

Figure 1. System Structure of Digital City Management System

\subsection{The Subsystems of Digital Intelligent City Management System}

1. Subsystem of wireless data acquisition

This subsystem is mainly used for the supervisor to report the information about the city management issues during their inspection in their own management unit mesh. The system based on mobile devices, using wireless communication network data transmission technology, relying the city components and events classification coding system and geo-coding system, completes the transmission of city management issues information in words, images, sounds and location information real-timely.

2. Subsystem of supervision center receives

This subsystem is designed for the supervision center of city management, employing personnel as supervision center operators. The information submitted by the "subsystem 
of wireless data acquisition" is passed to the working platform of the operators through the information service engines. And then, the operators receive, process and feedback various problems through the system to finish the collection, processing and filing of information. All the work provides collection and filing service of the city management issues for "the subsystem of collaboration" and ensures that problematical information can be accepted timely and accurately, and transferred to the command center.

\section{Subsystem of collaboration}

This subsystem, based on Browser/Server architecture, applying workflow and WebGIS technology, completes the specific transactions and information inquiries of each city management business through the browser. The subsystem of collaboration serves for the supervision center, the command center, various professional sectors, as well as leaders at all levels. The subsystem provides a workflow-based Gis for collaborative management, workflow processing, and supervision, it also offers the tools to share and query types of information resources of city management 10 r all kinds of users. Additional, based on different authority, it edits and queries geographic information, geo-information, city management components (events) information, supervision information, and so on. This system has realized the synergy office, the information synchronization and the exchange of information

\section{Subsystem of city components updating online}

According to the specification of components encoding, this subsystem provides specific access control modules to meet the needs of professional departments, who can update and maintain the data on the componentsthrough the IE browser.

\section{Subsystem of command and Gupervision on large-screen}

This subsystem services for supervision Center and command center, illustrating exactly the overall situation about the related map information, file information and other detailed information of the city management on the large screen. Similarly, the system is able to guery and present information of each community, supervisor and component and other individuas, and then get to hold of the overall situation of city management

6. Subsystem of comprehensive evaluation for city management

For the performance quantification and comprehensive evaluation, this subsystem makes a comprehensive analysis and calculates estimates on the information of regions, departments and positions in the database groups, generating performance-based evaluation results in accordance with the process, the main responsibility, work performance hormal specification and other evaluation models built-in the system.

\section{Subsystem of geo-coding}

Thi subsystem furnishes the subsystems of wireless data acquisition, collaboration, supervision and command on large-screen and others with geo-coding service, playing a role of describing, inquiring, and matching address.

8. Subsystem of application and maintenance

Responsible for the configuration, maintenance and management the whole system, applying the instrumentalization theory, this subsystem completes the flexible configuration of city management information, such as organizational structure, 
workflow, input form, output form, the use of map, inquiry and statistics. It can be configured to form the corresponding resource information, business rules and the definition of data manipulation using kinds of tools and components supplied by the system.

9. Subsystem of basic data resource management

This subsystem is used to realize the management, maintenance and expansion of spatial data resource, moreover, configure the functions of displaying, querying, editing and calculating spatial data.

10. Subsystem of data-exchanging and sharing system platform

Some information technologies such as mature XML, SOAP and Web Service can be used to establish an information-exchange platform, which includes the data exchange engine, remote data transmission and standard of exchange information and other core modules.

\section{Design Relevant System Interface of Digital Intelligent City Management System}

\subsection{Interface of Wireless Data Transmission}

Wireless data transmission mainly refers to the data transmission between wireless data acquisition system and database service. Using wireless cata collection tool, we can transmit the collected related information on event component, ineluding the types, relevant pictures, audio materials, location coordinates and other information of event (component) to the service by GPRS data transmission technology[4]

The data transmission between wireless terminal and service should support the HTTP hypertext transfer protocol and being able to achieve the information transmission in text, graphics, sound and image Cherefore, it should fit with the following requirements:

1. support HTTP hypertext transfer protocol;

2. support the technology of vireless data transmission;

3. suppopt data encryption:

4. the speed rate of wireles actual communication can't be less than $30 \mathrm{Kbps}$;

5. maintain stable network communication quality; the success rate of the transmission of information must be more than $99.9 \%$.

6. concurrentusers who exchange and transform data with the service should be able to guarantee the good running of system.

\subsection{Interface among the Subsystems of Wireless Data Acquisition, Calling Accepted and Collaboration}

The subsystem of wireless data acquisition installed in the wireless data collection tool is mainly applied to collect the information of event (component) and send the results to the subsystem of calling accepted through a wireless network. After the results have been filed, it will get to subsystem of the collaboration. The subsystem of calling accepted and collaboration have the same server, so it is easy to exchange and retrieval the data. In addition to what have been mentioned, the subsystem of collaboration will transmit data with subsystem of wireless data acquisition, which mainly occurs when the files of the event (component) have been treated. It requires some supervisors to verify if the case scene has been correctly handled. Therefore, on some node of the process, the subsystem of 
collaboration has to send the subsystem of wireless data acquisition the verification instructions which can reflect the relevant information of event (component) and location information and so on in the subsystem of wireless data. After the on-site verification in accordance with the relevant information, supervisors feed back the results into the nodes of subsystem of collaboration[5].

The data needs to exchange real-timely is divided into two types: one is information of the event (component) sent to the subsystem of calling accepted by city supervisors through subsystem of wireless data acquisition, the other is task information sent to the city supervisors by working platform of calling $\mathrm{s}$ based on the need of tasks. Task information includes:

1. information reported about the social work and information that needs to be confirmed by the city supervisors.

2.information assigned task number by the calling center according to the incident reports of city supervisors.

3. information fed back by city supervisors when they assist at the scene on the demand of calling center in accordance with the situation of handling of the ineident

In the light of two types of information mentioned aboye, the information server is mainly concerned with two things: firstly, receive incident information sent by the subsystem of wireless data acquisition, read data from database and write it into database of city management platform, and send message to working platform of calling center. Secondly, receive task message from working platform of calling center read data from database of city management platform and write it into the database of subsystem of wireless data acquisition, and send message to subsystem of wireless data acquisition. The message server plays a role as a data bridge between subsystem of wireless data acquisition and working platform of calling center, helping city supervisops and calling center to exchange message with each other real-timely.

Message server can also do some other things, such as, providing function of updating data initiatively, read database of gubsystem of wireless data acquisition from time to time, adding new event (component) to database of city management platform, sending event (component) message to working platform of calling center, noticing working platform of calling center that there have been new incident message to be dealt with.

\subsection{Interface among the Subsystems of Wireless Data Acquisition, Collaboration and Geo-Coding}

The subsystem of gea-coding is composed of geo-coding and geo-coded database. Assisted by geo-coding ergines, the subsystems of wireless data acquisition and collaboration can complete the function of rapid positioning when they quickly navigate to the site of the incident through the address information.

Speeding and efficient positioning of associated components in the city management issues depends on the geo-positioning engine provided by subsystem of geo-coding. Only when the received information quickly navigated on the map, the city management department can have a good understand of the location of the incident, the surrounding environment, traffic condition, and then have a command timely. Because of this, the geo-positioning engine of geo-coding should be fit with the following requirements:

1. Locate all the non-spatial socio-economic information collected and investigated (with the address) on the map exactly and establish the relationship between information of non-spatial and spatial accurately.

2. Lock the location of the incident quickly in the grid units of meters for the supervision center of the city management. 
3. Site the arbitrary input address string on some position in the grid units of meters and provide the coordinate information of the location.

4. Generate geo-coding strings in standard quickly for any spatial location.

In short, digital city management makes the achievement of changing city management from the blind to the precise, from the manual to the informational.

\section{Design of Digital Intelligent City Management Service Call Center}

\subsection{Designing Principles of Digital Intelligent City Management Service Call Center}

The construction of the Call Center System of digital city management will be based on a variety of national technical standards and operational requirements, and adopt solutions of internationally advanced customer service center. Internal application network uses client / server architecture. Seats terminals adopt a three-tier architecture, and using the related data of the existing management system and other system, we build a unified customer service system[6]. The realization of the system lies on the use of open standard-based information technology and the software, hardware products which are in line with the international industry standards. Software development adopts microsoft advanced software engineering methods MSF and the object-oriented structural design process. The systems construction principles are as follows:

\section{Criterion and standard}

According to the national and local norms and protocols of 《China's domestic telephone network signal Technical Specification (Temporary Provisions) Act》 and its added norms, Its communication protocols and interfaces comply with the international standards, and will be the mainstream in future.

\section{Advanced and maturity}

It uses the latest technologies in the field of call centers, and guarantees that the system will not make a large-scale restructuring under the reason of the technical backwardness since the construction of the system. Also it can maintain its advanced nature through upgrading the system, and extend their life cycle, as well as to ensure that advanced technology is stable, mature ,and support existing nultiple call function and network protocols.

\section{Security}

To prevent kinds of malicious or inadvertent hacker behavior and virus attacks, and to carry on the security checks and filter of External Access installation of firewall. Internal staff confirm, limited authorizes and check the host visit establishment, and prevent unauthorized personnel from misuse or carrying on the violative activities.

\section{Expansibility and compatibility}

The system adopts modular design and the principle of phased implementation. In addition to meeting various needs of the users, the system can realize the needs of expansion conveniently, and save a lot of time and money for investors; The system platform can accommodate existing or prospective new brands equipment, and ensure the full interoperability of heterogeneous systems. To meet the deployment of hardware and software flexibility and capacity requirements for the future hardware and software, we should consider the needs and upgrading capabilities of system scale expansion. 


\subsection{Systematical Topology Framework of Digital City Management Service Call Center}

The Systematical topology framework of digital city management service call center is shown in Figure 2, together with main hardware configuration.

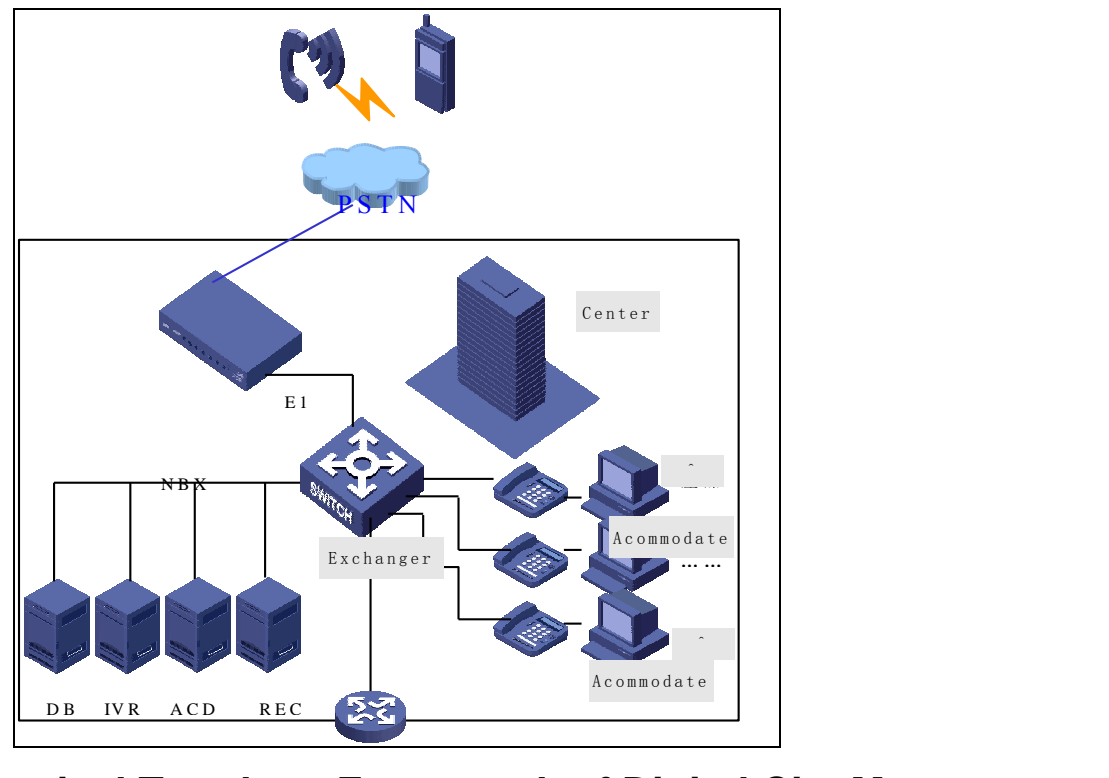

Figure 2. Systematical Topology Framework of Digital City Management Service Call Center

The core equipment is the NBX system of IP yoice over switching machine. The PSTNlines connect with the voice module port of NBX-system. And the Interactive Voice Response (IVR), Automatic Traffic distribution ( $(\mathrm{CD})$, interactive automated fax system (IFR), accommodate conversation recording (REC), as well as function of E-mail are carried out by the software installed in services. Meanwhile, we can send and receive messages by a mobilephone, which access to the SMS Gateway. The main features of this system are as follows: the core of Voice over IP, NBX system, offers the most simple network structure; provide various multi-channel meâns of communication (phone, fax, short message, mail); provide IP recording solution; provide standard operation-software port; protecting existing resources, and it can be linked with existing PBX telephone system.

The main hardware configurations are as follows: NBX V3000 Analog, SS3 NBX Chassis, EI card and impedance converter, 3101SPIP walkie-talkie, IP Phone Power Supply, special headphones of telephones and exchanger with the function of enantiomorphism port.

\subsection{Logic Structure of Digital Intelligent City Management Service Call Center Soltware}

Digital city management service call center software logic structure is shown in Figure 3. To more convenient and quicker deployment of call center system integration to enable endusers or developers through a simple configuration program or a spot of programme will be able to complete the entire implementation, the system provide Speed Call CTI-LINK middleware products based on Speed One-Station call center. CTI-LINK is modular program design based on COM technology, the flexibility and scalability is powerful. It provides a reliable and stable operation of the software platform to call center. It can be widely used in 
various sectors of the call center building, and effectively lowers the difficulty of the building and maintenance of call centers $[7,8]$.

CTI-LINK carries out many senior functions controlled by CTI call center, such as provide multi-media access, automated voice response, automated fax receipt and processing of external Call priority. Line statistics, Traffic Statistical management, accommodate recordings and other functions, CTI-LINK also provide free standard development interface to the application layer (system integration, Application developers) to facilitate third-party manufacturers to develop specific applications.

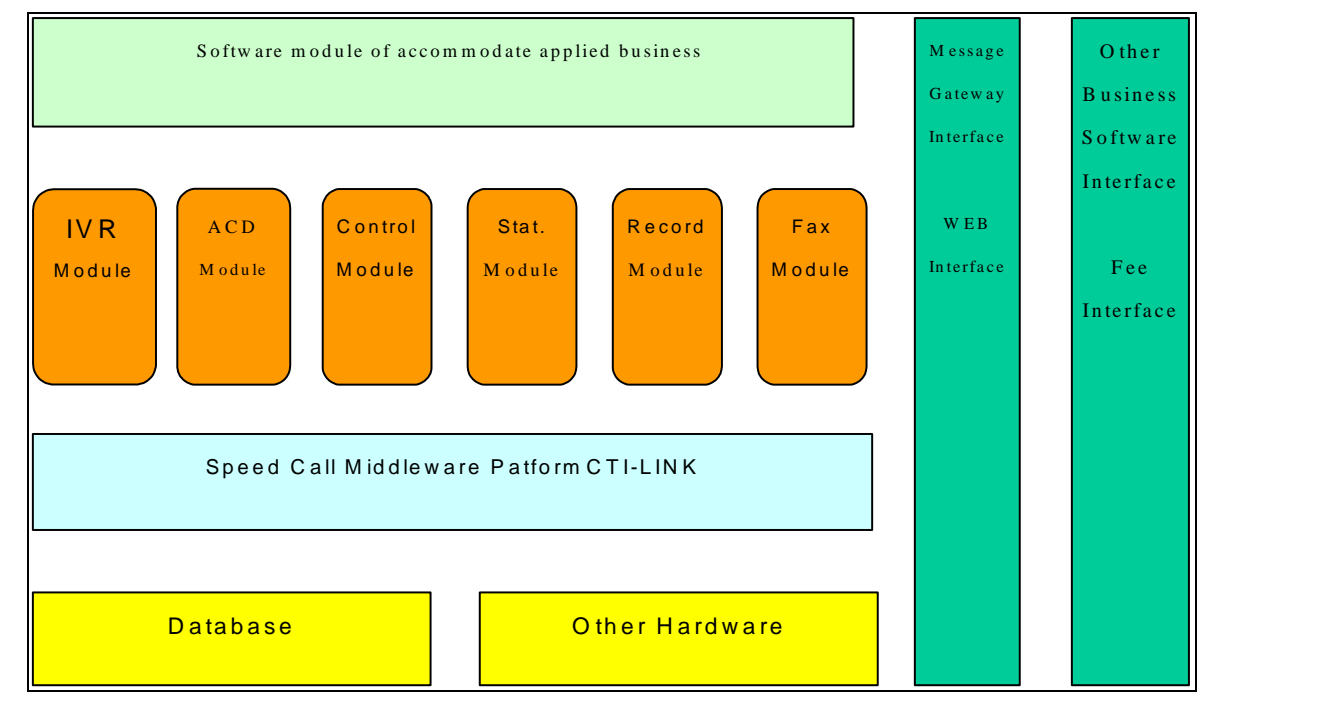

Figure 3. Digital City Management Service Call Center Software Logic

\section{Conclusion}

It is the development trend of city management to manage the city using the information technology. City management system provides information timely and accurately for the government to manage the social, solves the problems like information lagged and management passive, improyes the level and efficiency of city management as well as the management quality of government public service, helps enhance the comprehensive functions of cities and promote the sustainable development of city economics and society. The digital City service call center management system take the telephone as a center, attached to Infernet (WEB), the short message, the fax, the email (E-mail) and other modern means of jifformation, and use the computer formidable data processing and storage capacity, using advanced communications, computer network, computer integration and other advanced technology, combining the characteristics of municipal services, through mulimedia formats, such as voice, fax, Internet etc, completes interconnection between general residents and the municipal sectors all levels of networks unit. The system face the ordinary resident, provide convenience for the general residents understanding the various aspects of government policy and various departments serve function, help improve the efficiency and quality of municipal management service, change service mode, set up good service image of the government department, build and maintain good municipal administration management pattern. 


\section{Acknowledgments}

This paper was supported by NSFC (71272207, 71301150, U1304705), NSSF 13BGL061 and 10YJC630326 (Humanity and Social Science Foundation of Ministry of Education).

\section{References}

[1] Z. Yunfan and X. Yabin, "Based on CTI technology call center design and realization", Liaoning engineering institute journal, (2006).

[2] J. Bo, C. Ming and G. Guanqing, "Distributional and central unifies call center", Computer application, (2002).

[3] C. Wenhua, H. Xiaoping, C. Kai and B. Yingcai, "Based on VoIP technology virtual call center design and realization", Computer application and software, (2006).

[4] J. Geurt and G. Koole, "Managing uncertainty in call centers using Poisson mixtures" Applied Stochastic Models in Business and Industry, vol. 17, (2001), pp. 307-318.

[5] J. Luping, L. Kelu and T. Hua, "Based on three structures disperser -like call center system", Computer application, (2004).

[6] Y. Changhui, "Innovation System Framework of SME and Immovation Case Study", Proceedings of 2007 International Conference on Enterprise Engineering and Managemene Innovation, (2007), July 15-18; Hangzhou, China.

[7] S. Xuemin and Y. Changhui, "Constructing Service Call Center of Digital City Management System", Proceeding of 2nd International Conference on Intelligent Information Management Systems and Technology, (2007) June 21-26 Kantai, China.

[8] Y. Changhui, "Multi-Agent System of Evaluating and Selecting Supplier Basing on Quick Response Ability", Proceedings of the Sixth Wuhan International Conference on E-Business: Management Challenges in a Global World, (2007) May 16-20; Wuhan, China.

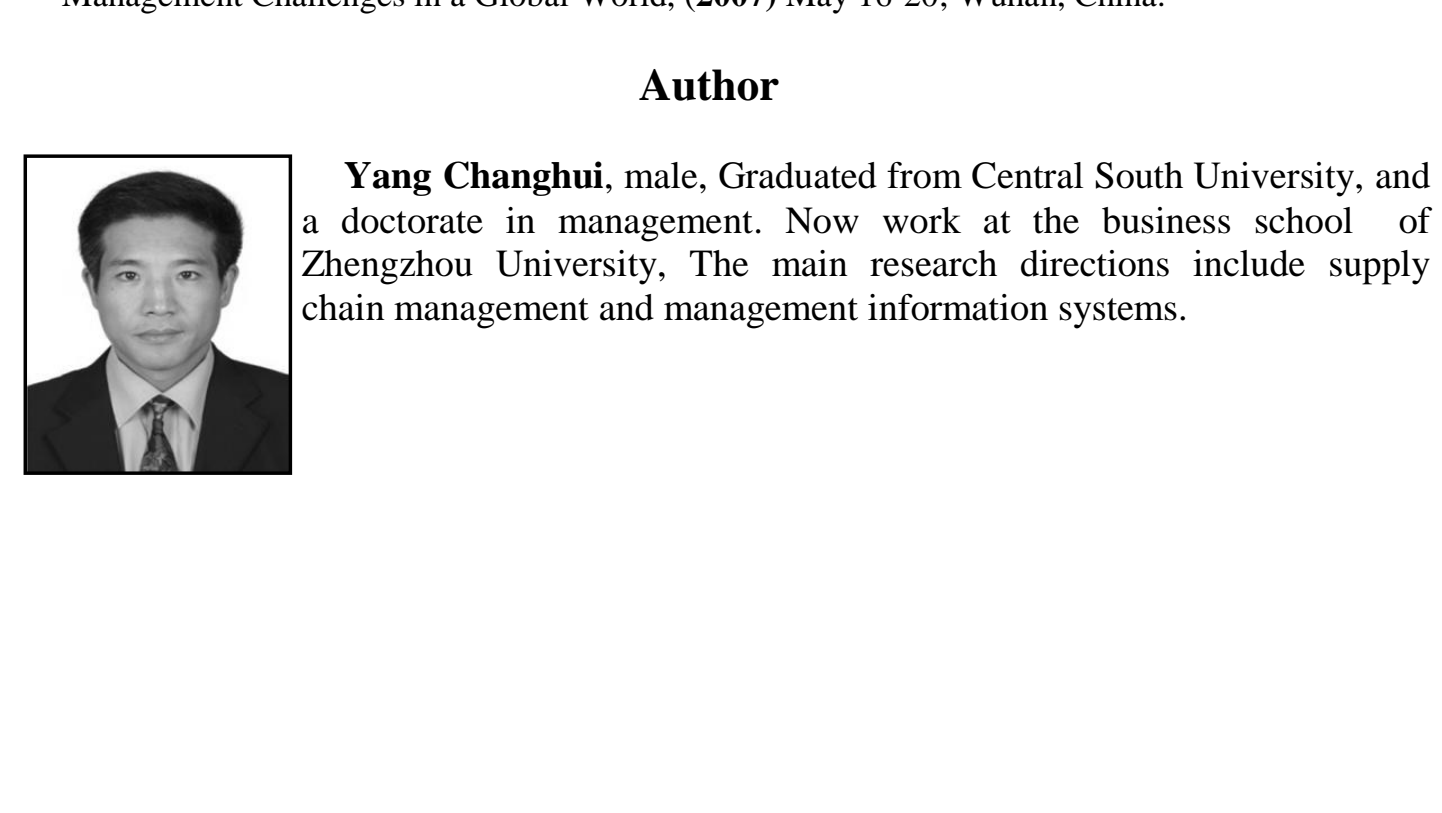


International Journal of Hybrid Information Technology

Vol.7, No.5 (2014)

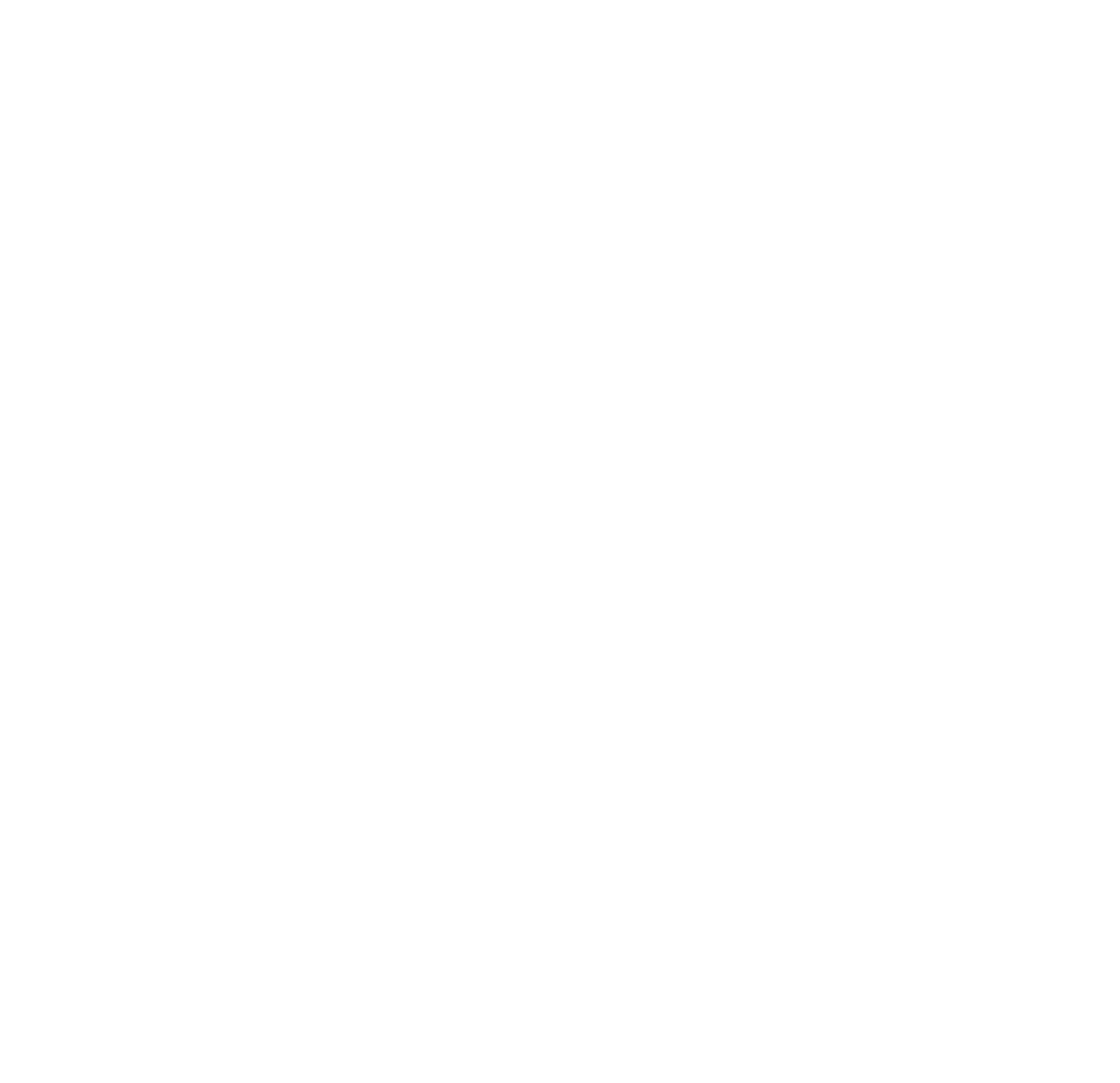

